\title{
Research on Wireless Sensor Actuator Distribution Algorithm Meeting Maximum Request Expectation
}

\author{
https://doi.org/10.3991/ijoe.v13i04.6584 \\ Xuejun Jiang \\ Shenyang Ligong University, Shenyang, Liaoning, P.R.China \\ Wenbo Zhang* \\ Shenyang Ligong University, Shenyang, Liaoning, P.R.China \\ 1159365437 @qq. com \\ Lincong Zhang \\ Shenyang Ligong University, Shenyang, Liaoning, P.R.China \\ Xiaobo Tan \\ Shenyang Ligong University, Shenyang, Liaoning, P.R.China
}

\begin{abstract}
In wireless sensor actuator networks, actuator nodes are very important to network coverage for wireless sensor, So according to the characteristics that wireless communication is easy to be affected by the environment, in this wireless sensor actuator network the actuator nodes are abstracted to be service providers and the sensor nodes are abstracted to be service consumers, Furthermore, the messages transferred from actuator nodes to sensor nodes are abstracted to be service requests and the coverage of sensor nodes by actuator nodes is service providing, Moreover, the service faulty ratio of actuator nodes is defined in this paper, Based on that, an actuator node coverage algorithm that satisfies the maximum request expectation is put forwarded, Its goal is to solve the mathematical expectation of maximizing the number of service requests with fixed number of sensor nodes and actuator nodes, So the optimal position of actuator nodes could be identified, The theoretic proof and simulation result show that this algorithm is more efficient compared with similar algorithms.
\end{abstract}

Keywords-wireless sensor actuator network; actuator distribution algorithm; mathematical expectation; maximum request expectation

\section{Introduction}

\subsection{Background and Motivation}

As one of intelligent networks with the function of interaction with the environment, Wireless Sensor Actuator Network (WSAN) brings the actuator nodes 
into the traditional wireless sensor network, WSAN has prospects of more extensive application in monitoring and processing of emergency, especially in high risk patient monitoring, forest fire detection, etc, [1] Real time is the most important features of WSAN because the perceived events must be carried out promptly and accurately, Therefore, in applications of an ideal WSAN, the actuator node should cover all the sensor nodes, such perceived events can be handled directly and timely, In practice, the deployment of large number of sensor nodes are scattered randomly by plane, However, the actuator node is with sufficient energy, rich resources, high cost and limited numbers, Therefore, researches on how to arrange actuator nodes reasonably according to the distribution of sensor nodes, will be beneficial to improve the efficiency of WSAN and reduce the cost[2],

At present, a lot of research is directed at the optimization deployment of large scale WSN nodes, studies on the deployment of actuator nodes in WSAN is less, Due to the differences between WSAN and WSN, unlike the main problem in WSN deployment is to deal with the maximum coverage of sensors in sensing area, the main solution in WSAN deployment is to realize the maximum coverage of actuator nodes for sensors by reasonable deployment of actuator nodes, The existing WSN coverage algorithm is difficult to applicate directly in WSAN, Therefore, for the characteristics of WSAN, this paper abstracts the actuator nodes as service nodes, and sensor nodes as user nodes, namely the actuator nodes provide service by covering the sensor nodes, Although a lot of research has been developed in the field of coverage of actuator nodes for sensor nodes, but the current study mainly focuses on how to make the actuator nodes cover as many sensor nodes as more[3], However, in WSAN, the heat of each sensor node is different, That is, due to the different location of each sensor node, different sensor nodes receive different sensory information, so the number of coverage requests for the actuator nodes is also different[4], Therefore, in the light of the differences between request numbers of each sensor node and the characteristics of wireless network communication, the paper proposes WASN deployment algorithm meeting the maximum request expectation[5].

\subsection{Related work}

Recently a lot of progress has been achieved in WSAN[6] with the development of intelligent sensors and actuators, Inspired by the molecular repulsive force in the micro-world, the actuator node can be uniformly distributed in the monitored area so as to make the maximum coverage, and actuator nodes in the range of the mutual communication[7], However, the actuator nodes are arranged by triangular mesh, which means un-coverage areas exist in monitoring area, i,e, blind zone[8], The sensor nodes deployed in blind zone cannot play any role[9].

An maximum coverage area algorithm (DA2MC) based on distributed arrangement of actuator nodes is proposed in [10], By dividing the monitored area into several hexagon regions, actuator nodes are arranged in the center of each hexagon and the nodes in each hexagon area form a cluster through selforganization, The experimental results shows that the whole monitored area can be covered completely, and the number of used actuator nodes is the least. 
For example, there are proposals that prioritize geographic area coverage as well as barrier coverage, rather than the coverage of discrete targets, and allow for network nodes to move after deployment [11], In [12], a maximum covers algorithm using mixed integer programming (MC-MIP) was proposed, Based on the output of MCMIP, nodes are organized into disjoint set covers (DSC) which are activated successively, In [13], a greedy algorithm designed for maximum set covers (MSCGreedy) was proposed, Cover sets generated from MSCGreedy are not required to be disjoint and are allowed to operate in different time intervals, Compared with MC-MIP, MSC-Greedy produces better results in terms of network lifetime, since the solution space of DSC problem is included in the solution space of the maximum set cover (MSC) problem, However, current researches on sensor nodes deployment mainly focus on ensuring sensing areas connectivity through optimizing sensing coverage probability (CP), For example, [14] devised the nodes deployed model based on Gaussian distribution and concluded that sensing CP was improved with the increase of standard deviation of the deployed errors; [15] introduced missing probability to obtain optimal number of deployed sensor nodes and deployment according to the terrain model and the deployed precision, and reformed the algorithmproposed in [16], which optimized CP to improve deployed efficiency in terms of uncertainty of sensing probability.

\subsection{Paper Organization}

The rest of the paper is organized as follows, Section 2 establish the actuator distribution algorithm meeting maximum request expect, Section 3 gives the basic mathematical theories and their proof, Section 4 presents the simulation results, Section 5 concludes the paper,

\section{Actuator distribution algorithm meeting maximum request expectation (ADAMMRE)}

The proposed algorithm (ADAMMRE) is built based on the following assumptions:

(1) both all sensor nodes and actuator nodes can finish a single task;

(2) All sensor nodes are facilitated with a GPS module, and the locations have been fixed;

(3) All nodes deployed randomly in sensing area have a unique Identification (ID);

(4) All nodes cannot move freely;

(5) The communication links between nodes are bidirectional symmetric;

(6) There is some relation of service between sensor nodes and actuator nodes, that is, the actuator nodes provide service for the sensor nodes.

Assume that there are $\mathrm{N}$ sensor nodes and $\mathrm{M}$ actuator nodes in WASN, and the $\mathrm{M}$ actuator nodes provide service for the $\mathrm{N}$ sensor nodes and maximize the number of service request $Y$, sensor nodes and actuator node can be at the same position. 
$h_{k}$ denotes the request times of sensor node $k \in[1, N]$;

$y_{k i}$ denotes the semaphore, if node $i$ is overlapped by actuator $k$, then $y_{k i}=1$; else $y_{k i}=0$, If one or more actuators exist in the location of node $i, i \in[1, M]$, then $x_{i}=1$; else $x_{i}=0$, Service radium of the actuator node is $D$, the distance between sensor node $i$ and actuator node $k$ is $d_{k i}$, if $d_{k i}<D$, then sensor node $i$ will be overlapped by actuator node $k$, coverage $_{k i}=1$; else actuator node $k$, cannot overlap sensor node $i$, then coverage $_{k i}=0$.

In conclusion,

$$
\begin{gathered}
y_{k}-\sum_{i=1}^{N} \text { coverag }_{k i} x_{i} \leq 0 \quad k=1,2 \mathrm{~L}, N \\
\sum_{i=1}^{N} X_{i} \leq M
\end{gathered}
$$

The number of actual deployed actuator nodes should be smaller than that of actual existing ones, In addition, when any actuator node exists at the location of node $i$ and the distance between it and sensor node is not larger than its service radius, the sensor node can be served,

Furtherly, assumed that failure probability of actuator node providing service to sensor node is $p$, failure probabilities of all actuator nodes are identical, and there is no coupling relationship between the failure probabilities of each node, then the number of actuator nodes $T$ that can provide service normally should be obey binomial distribution, i,e,, $P\{T=j\}=C_{M}^{j}(1-p)^{j} p^{M-j}$, Thus, the probability of $m$ actuator nodes serving sensor node $k$ is,

$P$ \{the probability of node $\mathrm{k}$ is overlapped by actuator nodes

$=1-P\{\mathrm{~m}$ actuator nodes fail simultaneously $\}$

$=1-p^{m}$

Therefore, assume that random variable $H_{k, m}$ denotes the request number of node $k$ covered by $\mathrm{m}$ actuator nodes,

$$
\begin{aligned}
& H_{k, m}= \begin{cases}h_{k} & \text { the probability is } 1-p^{\mathrm{m}} \\
0 & \text { the probability is } p^{\mathrm{m}}\end{cases} \\
& E\left(H_{k, m}\right)=h_{k}\left(1-p^{m}\right), \quad \forall k, m
\end{aligned}
$$

When the number of actuator nodes covering sensor node $\mathrm{k}$ changes from $\mathrm{m}-1$ to $\mathrm{m}$ , the increment of request times expect of node $\mathrm{k}$ can be represented as Eq,(5),

$$
\begin{gathered}
\Delta E\left(H_{k, m}\right)=E\left(H_{k, m}\right)-E\left(H_{k, m-1}\right)=h_{k} p^{m-1}(1-p) \\
m=1,2, \mathrm{~L}, M \quad(5)
\end{gathered}
$$


Consequently, the maximum service request number is $\sum_{k=1}^{N} \sum_{j=1}^{M}(1-p) p^{m-1} h_{k} y_{j k}$.

Considering the characteristics of the different residual energy of nodes in WSN, the service failure probability of each actuator node is not equal, This paper further defines the service failure probability $p_{i}$ of actuator node $i$ as Eq, (6).

$$
p_{i}=p-\alpha p+\alpha\left(1-E r_{i} / E i_{i}\right)
$$

So the probability of both $\mathrm{m}$ actuator nodes cannot provide service is $\prod_{i=1}^{m} p_{i}$.

$P\{$ the success probability of node $i$ is served by $m$ actuator nodes $\}=1-p^{m}=1-\prod_{i=1}^{m} p_{i}$

According to Eq, (4):

$$
\begin{aligned}
& \mathrm{E}\left(H_{k, m}\right)=h_{k}\left(1-p^{m}\right)=h_{k}\left(1-\prod_{j=1}^{m} p_{j}\right) \\
& \Delta E\left(H_{k, m}\right)=E\left(H_{k, m}\right)-E\left(H_{k, m-1}\right) \\
& =h_{k}\left(1-\prod_{i=1}^{m} p_{i}\right)-h_{k}\left(1-\prod_{i=1}^{m-1} p_{i}\right) \\
& =h_{k}\left(1-p_{m}\right) \prod_{i=1}^{m-1} p_{i} \\
& \operatorname{Max}, Y=\sum_{k=1}^{N} \sum_{j=1}^{M} h_{k} y_{j k}\left(1-p_{j}\right) \prod_{i=1}^{j-1} p_{i}
\end{aligned}
$$

St, $p_{\mathrm{i}}=p-\alpha p+\alpha\left(1-E r_{\mathrm{i}} / E i_{\mathrm{i}}\right)$

Where $y_{j k}= \begin{cases}1 & \text { if node } j \text { is covered by actuator node } k \\ 0 & \text { else }\end{cases}$

$p \quad$ denotes the average failure probability of nodes.

$\alpha$ denotes the adjustment factor, adjusts the impact degree of energy in clustering algorithm.

$E i_{\mathrm{i}} \quad$ denotes the initial energy of node $i$.

$E r_{\mathrm{i}}$ denotes the residual energy of node $i$,

$M \quad$ denotes the number of actuator nodes.

$N$ denotes the number of sensor nodes.

Where $\alpha$ is the impact factor of node energy on service failure probability, $p_{i}$ is composed of network average failure probability $p$ and node's energy factor $\left(1-E r_{\mathrm{i}} / E i_{\mathrm{i}}\right)$, the percentage of network average failure $p$ in $p_{i}$ decreases with the increase of $\alpha$, Conversely, the percentage of node's energy factor increases with the increase of $\alpha$, When $\alpha=0, p_{\mathrm{i}}=p$, means that service failure probabilities of all current actuator nodes are the same, When $\alpha=1, p_{\mathrm{i}}=1-E r_{i} / E i_{i}$, the clustering 
algorithm is affected only by node's energy factor, In fact, the value of $\alpha$ is determined by the current network communicating environment and consumption rates of node energy,

\section{Theoretical analysis and validation}

[Theorem 1] If the total number of actuator nodes and request numbers of each sensor node are fixed, the lower service failure probability of each actuator node makes more total network request number,

Proof: Assume that the total numbers of network actuator nodes and sensor nodes are $M$ and $N$, respectively, the service failure probabilities of each actuator node are $p_{i}$ and $p_{i}^{\prime}(i=1,2, \mathrm{~L} M)$, respectively, and $p_{i}<p_{i}^{\prime}$, the request number of sensor nodes in the whole network are $F$ and $F^{\prime}$, then

$$
\begin{aligned}
& F=\sum_{k=1}^{N} h_{k}\left(1-\prod_{i=1}^{M} p_{i}\right) \text {,where node } \mathrm{k} \text { is covered by } \mathrm{R} \text { actuator nodes, } \\
& F^{\prime}=\sum_{k=1}^{N} h_{k}\left(1-\prod_{i=1}^{M} p_{i}^{\prime}\right)
\end{aligned}
$$

For any $i(i=1,2, \ldots, M), p_{i}<p_{i}^{\prime}$.

$$
\begin{aligned}
& F-F^{\prime}=\sum_{k=1}^{N} h_{k}\left(1-\prod_{i=1}^{R} p_{i}\right)-\sum_{k=1}^{N} h_{k}\left(1-\prod_{i=1}^{R} p_{i}^{\prime}\right) \\
&=\sum_{k=1}^{N} h_{k}-\sum_{k=1}^{N} h_{k}\left(\prod_{i=1}^{R} p_{i}\right)-\sum_{k=1}^{N} h_{k}+\sum_{k=1}^{N} h_{k}\left(\prod_{i=1}^{R} p_{i}^{\prime}\right) \\
&=\sum_{k=1}^{N} h_{k}\left(\prod_{i=1}^{R} p_{i}^{\prime}\right)-\sum_{k=1}^{N} h_{k}\left(\prod_{i=1}^{R} p_{i}\right) \\
&=\sum_{k=1}^{N} h_{k}\left(\prod_{i=1}^{R} p_{i}^{\prime}-\prod_{i=1}^{R} p_{i}\right) \\
& Q \quad P_{i}^{\prime}>P_{i} \\
& \therefore \quad \prod_{i=1}^{R} p_{i}^{\prime}>\prod_{i=1}^{R} p_{i} \\
& \therefore \quad F-F^{\prime}>0
\end{aligned}
$$

So the theorem is proved.

[Lemma 1] If the average network failure probability and adjustment factor $\alpha$ are fixed, the larger residual energy of actuator nodes serves more network requests.

By definition, service failure probability of actuator node $i$ is $p_{\mathrm{i}}=p-\alpha p+\alpha\left(1-E r_{\mathrm{i}} / E i_{\mathrm{i}}\right)$, If the residual energy of actuator node $(i=1,2, \mathrm{~L} M)$ and the average network failure probability $p$ and $\alpha$ do not change, then $p_{i}<p_{i}^{\prime}$, By theorem $1, F>F^{\prime}$, so the Lemma 1 has been proved. 
[Lemma 2] If the residual energy of actuator nodes and adjustment factor $\alpha$ are fixed, the lower average network failure probability leads to more network requests that can be served.

By definition, service failure probability of actuator node $i$ is $p_{\mathrm{i}}=p-\alpha p+\alpha\left(1-E r_{\mathrm{i}} / E i_{\mathrm{i}}\right)$, If the residual energy of actuator node $E r_{i}$ and $\alpha$ do not change, and average network failure probability $p<p^{\prime}$, then $p_{i}<p_{i}^{\prime}$, By theorem $1, F>F^{\prime}$, so the Lemma 2 has been proved.

[Lemma 3] If the average network failure probability and the residual energy of actuator nodes are fixed, the larger the adjustment factor $\alpha$, the more network requests that can be served,

By definition, service failure probability of actuator node $i$ is $p_{\mathrm{i}}=p-\alpha p+\alpha\left(1-E r_{\mathrm{i}} / E i_{\mathrm{i}}\right)$, If the residual energy of actuator node $E r_{i}$ and the average network failure probability $p$ do not change, the adjustment factor $\alpha>\alpha^{\prime}$, then $p_{i}<p_{i}^{\prime}$, By theorem $1, F>F^{\prime}$, so the Lemma 3 has been proved.

[Theorem 2] If the service coverage failure probability of existing actuator nodes keeps fixed, with the increase of the number of actuator nodes, the total service requests also increases.

Proof: Assume that the total numbers of network sensor nodes is $N$, the number of actuator nodes changes from $m_{1}$ to $m_{2}$, When the number of actuator nodes is $m_{1}$, the set of service coverage numbers of actuator nodes is $Y=\left\{y_{1}, y_{2}, \ldots \ldots y_{N}\right\}$, and the optimal deployment solution of actuator nodes is $L=\left\{l(1), l(2), \ldots \ldots, l\left(\mathrm{~m}_{1}\right)\right\}$, When the number of actuator nodes is $m_{2}$, the set of service coverage numbers of actuator nodes is $Y^{\prime}=\left\{\mathrm{y}_{1}^{\prime}, \mathrm{y}_{2}^{\prime}, \ldots . . \mathrm{y}_{N}^{\prime}\right\}$, and the optimal deployment solution of actuator nodes is $L^{\prime}=\left\{l^{\prime}(1), l^{\prime}(2), \ldots . . ., l^{\prime}\left(m_{2}\right)\right\}$.

Because of $m_{1}<m_{2}, m_{2}>m_{1}+1$ or $m_{2}=m_{1}+1$ is right and can be discussed by the following two situations.

(1) $m_{2}=m_{1}+1$

Assume that $l^{\prime}(i)=l(i), i=1,2, \ldots \ldots m_{1}$, and $l^{\prime}\left(m_{2}\right)$ is any one of the candidate locations,

Due to the emergence of the $m_{2}$ actuator node, the covering relations between some of the sensor nodes and actuator nodes may change, the total number of service requests provided is likely to change.

(i) If the emergence of the $m_{2}$ actuator node does not change the covering relations to sensor nodes because of the join of the $m_{2}$ actuator node, then for any sensor node $i, y_{i}^{\prime}=y_{i}(i=1,2, \mathrm{~K} \mathrm{~N}), \mathrm{i}, \mathrm{e}, Y=Y^{\prime}$, Thus, $\sum_{k=1}^{N} h_{k}\left(1-\prod_{i=1}^{y_{i}^{\prime}} p_{i}\right)=\sum_{k=1}^{N} h_{k}\left(1-\prod_{i=1}^{y_{i}} p_{i}\right)$ is hold, further $F\left(m_{1}\right)=F\left(m_{2}\right)$. 
(ii) If the covered times of existing sensor node $y_{i}^{\prime}>y_{i}$, then by definition, the $m_{2}$ actuator node coverage the sensor node $i$, and the set of service coverage times of the whole network changes, So.

$$
\begin{aligned}
& 1-\prod_{j=1}^{y_{i}^{\prime}} p_{j}>1-\prod_{j=1}^{y_{i}} p_{j} \\
& \Rightarrow h_{k}\left(1-\prod_{j=1}^{y_{i}^{\prime}} p_{j}\right)>h_{k}\left(1-\prod_{j=1}^{y_{i}} p_{j}\right) \\
& \Rightarrow \sum_{k=1}^{N} h_{k}\left(1-\prod_{j=1}^{y_{i}^{\prime}} p_{j}\right)>\sum_{k=1}^{N} h_{k}\left(1-\prod_{j=1}^{y_{i}} p_{j}\right)
\end{aligned}
$$

According to above reasoning, it is easy to achieve the conclusion $F\left(m_{1}\right)<F\left(m_{2}\right)$.

Combing (i) and (ii), we can see that under the assumption of (1), $F\left(m_{1}\right) \leq F\left(m_{2}\right)$ is hold.

(2) $m_{2}>m_{1}+1$

Due to $m_{2}>m_{1}+1$, there must be one $m^{\prime}$ which enables $m^{\prime}=m_{1}+1$ and $m^{\prime}<m_{2}$, By the proof of (1), we can see that $F\left(m_{1}\right) \leq F\left(m^{\prime}\right)$, As a result, if $m_{2}>m_{1}+1$, then the maximum number of service coverage requests satisfies $F\left(m_{1}\right)<F\left(m_{2}\right)$.

Synthesizing the proof of (1) and (2), if $m_{1}<m_{2}$ then $F\left(m_{1}\right) \leq F\left(m_{2}\right)$.

[Theorem 3] If service coverage radius of actuator nodes increases, the maximum number of service coverage requests also increases.

Proof: Assume that there are $N$ sensor nodes and $M$ actuator nodes in WSN, When the service coverage radius is $C R_{1}$, the set of coverage times is $C N=\left\{c n_{1}, c n_{2}, \ldots . . c n_{N}\right\}$ and the locations of actuator nodes are $L^{\prime}=\{l(1), l(2), \mathrm{L}, l(n)\}$, When the service coverage radius increases to $C R_{2}$, the set of service coverage times is $C N^{\prime}=\left\{c n_{1}^{\prime}, c n_{2}^{\prime}, \ldots . . c n_{N}^{\prime}\right\}$ and the locations of actuator nodes are $L^{\prime}=\left\{l^{\prime}(1), l^{\prime}(2), \mathrm{L}, l^{\prime}(n)\right\}$, Since the service coverage radius of actuator nodes changes from $C R_{1}$ up to $C R_{2}$, it will result in the change of the service covered situation of the sensor nodes in the network, Therefore, we should analyze the following two cases.

(1) If there is no sensor node $i$ in the network of which coverage situation changes with the increase of service coverage radius, then the set of served coverage times of the whole network will not change, i,e, $C N=C N^{\prime}$, Consequently, the successfully served probability of each sensor node within service coverage radius will not change, $\mathrm{i}, \mathrm{e}, 1-1-\prod_{i=1}^{c n_{i}^{\prime}} p_{i}=1-\prod_{i=1}^{c n_{i}} p_{i}, k=1,2, \mathrm{~L}, N$, Thus sum the number of covered requests of each sensor node in the network, $\sum_{k=1}^{N} h_{k}\left(1-\prod_{i=1}^{c n_{i}^{\prime}} p_{i}\right)=\sum_{k=1}^{N} h_{k}\left(1-\prod_{i=1}^{c n_{i}} p_{i}\right)$, i,e, $F\left(C R_{1}\right)=F\left(C R_{2}\right)$. 
(2) If there is any sensor node $k$ in the network, the number of its served coverage changes, then the set of served coverage times will also change, i,e, $C N \neq C N^{\prime}$, According to the description of model, not only the set of served coverage times will change with the increase of the service coverage radius, but also the optimal location set of actuator nodes will be affected.

(a) If the optimal location set does not change with the increase of the service coverage radius, then $L^{\prime}=L$, Due to $C N \neq C N^{\prime}$, by the definition of the model, there is at least one sensor node $k$ which enables $c n_{k}^{\prime}>c n_{k}$, Thus, the probability of this node can be served successfully within the service coverage radius satisfies $1-\prod_{i=1}^{c n_{i}^{\prime}} p_{i}>1-\prod_{i=1}^{c n_{i}} p_{i}$, Then multiply both sides of inequality by the node's request numbers $h_{k}$ and then sum them, so $\sum_{k=1}^{N} h_{k}\left(1-\prod_{i=1}^{c n_{i}^{\prime}} p_{i}\right)>\sum_{k=1}^{N} h_{k}\left(1-\prod_{i=1}^{c n_{i}} p_{i}\right)$ is hold, that is, the maximum request number covered by service satisfies $F\left(C R_{1}\right)<F\left(C R_{2}\right)$ when the coverage radiuses are $C R_{1}$ and $C R_{2}$.

(b) If the optimal location set changes because of the increase of coverage radius, then $L^{\prime} \neq L$, Because $L^{\prime}$ is the optimal location solution when the coverage radius is $C R_{1}$, the request number covered by service of the other location solutions with the coverage radius $C R_{2}$ is not larger than the maximum request number covered by service $F\left(C R_{2}\right)$ under $L^{\prime}$, That is, the request number covered by service of location solution $L$ satisfies $F^{\prime}\left(C R_{2}\right)<F\left(C R_{2}\right)$ when the coverage radius is $C R_{2}$, Simultaneously, according to the proof of (a), since both the corresponding location solutions of $F\left(C R_{1}\right)$ and $F^{\prime}\left(C R_{2}\right)$ are $L$ and the coverage radius $C R_{1}<C R_{2}$, so $F\left(C R_{1}\right)<F^{\prime}\left(C R_{2}\right)$ is hold, Combing these two points, with the current assumptions, $F\left(C R_{1}\right)<F\left(C R_{2}\right)$ is hold.

It can be seen that, when the set of coverage times changes, $F\left(C R_{1}\right) \leq F\left(C R_{2}\right)$ is hold.

\section{$4 \quad$ Simulation and analysis}

In order to verify the algorithm performances, the paper carries on the simulation by using the simulation tool MATLAB 7,0, The simulation scenario is a $500 \mathrm{~m} \times 500 \mathrm{~m}$ square area, in which 300 sensor nodes are distributed randomly, The number, the initial location and the corresponding radius (coverage range) of actuator nodes are adjustable parameters in the simulation.

With the same number of requests of all sensor nodes, the service coverage of the actuator nodes for sensor nodes is shown in Figure 1, As we can see that, the service coverage for sensor nodes of the proposed algorithm is obviously superior to the method that randomly deploys the actuator nodes, Compared with the MRT-VOR 
algorithm, AMRWOD algorithm can provide higher service coverage, has better performance, This is because that the MRT-VOR algorithm adopts distributed node deployment protocol, carries out movement competition by movement rounds and movement authority, which leads to energy consumption of actuator node in each round of movement, and so extends execution time of the algorithm, While AMRWOD algorithm taking use of the capability of computing and communication of base station for centralized deployment, and thus the algorithm has faster execution speed and shorter convergence time.

With the different numbers of requests of each sensor nodes, taking the sensor node in hot spot for example, its sensing amount of data is large, so more actuator nodes are needed to provide more service coverage, In this paper, we assume that the minimum and the maximum request number of sensor nodes are 1 and 10, respectively, and the numbers of requests of each sensor node are not exactly the same, The simulation results are shown in Figure 2, Analysis shows that, for the sensor nodes with small number of service coverage requests, the service coverage rate is between $85-95 \%$, and for the sensor nodes with large number of service coverage requests, the actuator nodes can reach to complete coverage.

With unchanged service coverage failure probability of existing actuator nodes, the times of sensor nodes covered by service also increase with the increase of the number of actuator node, as shown in Figure 3.

With unchanged service coverage failure probability of existing actuator nodes, the times of sensor nodes covered by service also increase with the increase of coverage radius of actuator node.

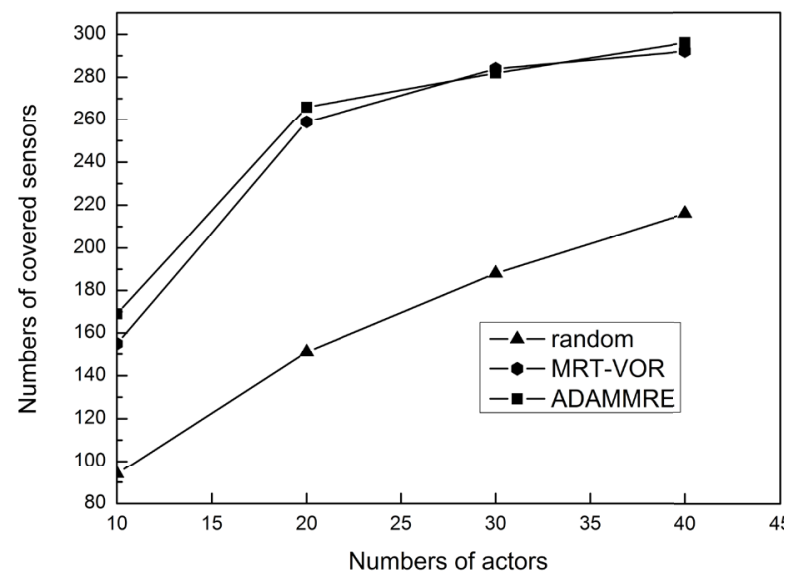

Fig. 1. Comparison of coverage number of sensor by different number of actuators 


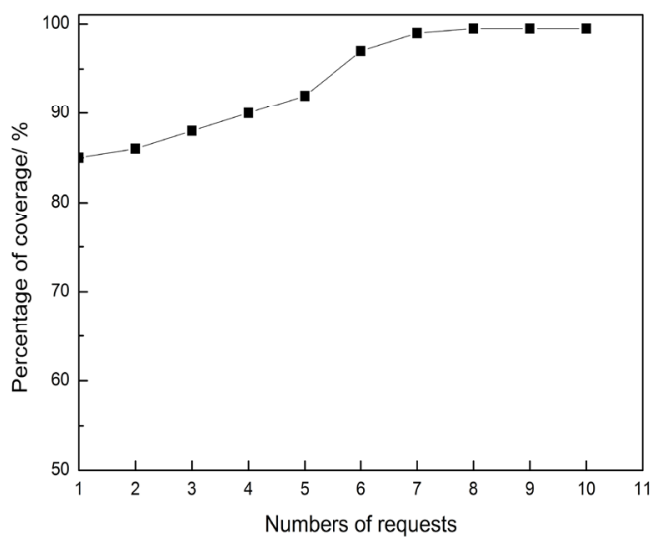

Fig. 2. Relationship of number of requests with percentage of coverage

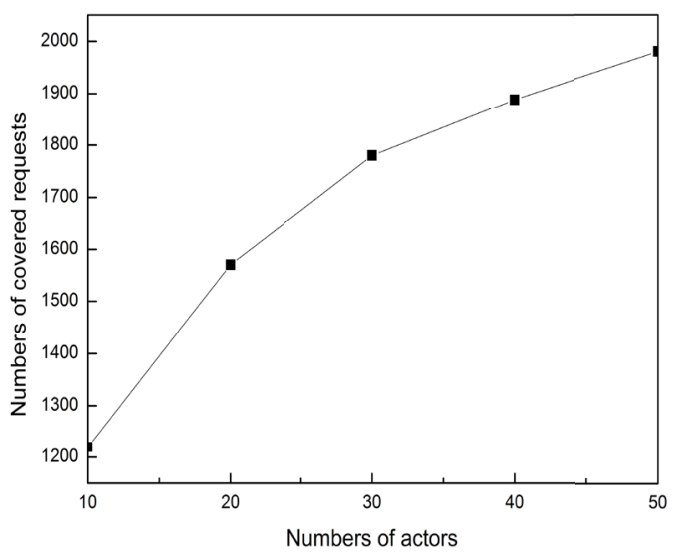

Fig. 3. Relationship between actuator nodes and service coverage times

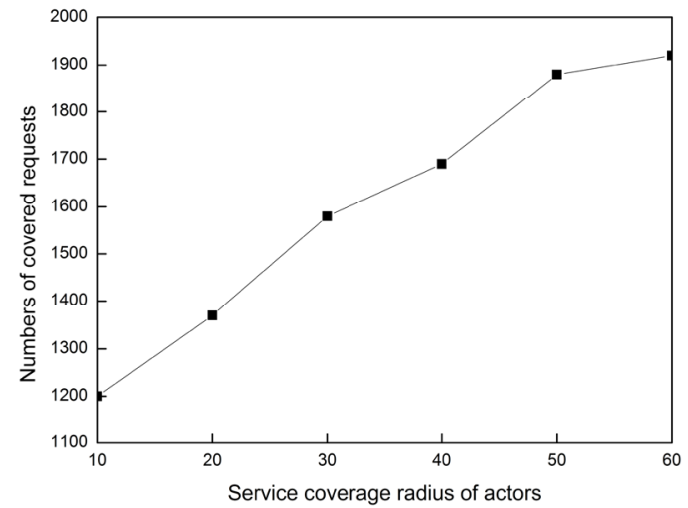

Fig. 4. Relationship between service coverage radius and service request numbers 


\section{Conclusion}

In wireless sensor actuator network, the actuator nodes distribution algorithm is vital for coverage of sensors, So in terms of the wireless communication environment, an actuator distribution algorithm meeting maximum request expectation is put forward, The theoretical proof showed that this new algorithm is suitable for wireless sensor actuator networks, Furthermore the simulation results show that more requests could be responded and more sensors could be covered and be served.

\section{Aknowledgement}

This paper is sponsored by National Natural Science Foundation of China (61501308), Liaoning province innovation group project (LT2011005), the Shenyang Ligong University Computer Science and Technology Key Discipline Open Foundation(2012,2013), Liaoning fourth batch of distinguished professor project(2014) and Liaoning BaiQianWan Talents Program.

\section{$7 \quad$ References}

[1] $S, F, L i$, Wireless sensor actuator network for light monitoring and control application, Proceedings of the consumer communications and networking conference, Las Vegas, 2006, pp, 974-978.

[2] $W, H u, N, B u l u s u, S, J h a$, A communication paradigm for hybrid sensor/actuator networks, Proceedings of PIMRC, Barcelona, 2004, 201-205.

[3] $H$ Wang, $X X u$, Wireless Sensor Network in the Photovoltaic Power Generation Monitoring System, Telkomnika Indonesian Journal of Electrical Engineering, vol,11, no, 8, 2013, pp, 4774-4779.

[4] K, Morita, K, Ozaki, et al, Evaluation of reliable data transmission protocol in wireless sensor-actuator network, International Conference on Advanced Information Networking and Applications Workshops (AINAW), Niagara Falls, vol. 2, 2007, pp. 713-718, https://doi.org/10.1109/ainaw.2007.177

[5] J, Wan, C, Zou, S, Ullah, C F, Lai, M, Zhou, and X, Wang, Cloud-Enabled wireless body area networks for pervasive healthcare, IEEE Network, 2013, vol,27, no,5, pp, 56-61.

[6] Vipin Pal, Yogita, Girdhari singh, R P Yadav, Effect of heterogeneous nodes lacation on the performance of clustering algorithm for wireless sensor networks, International Conference on Recent Trends in Computing (ICRTC 2015), Ghaziabad, 2015: 1042-1048.

[7] Mustafa Ilhan Akbas, Damla Turgut, Lightweight routing with dynamic interests in wireless sensor and actor networks, Ad Hoc Networks, vol. 11, 2013, pp, 2313-2328.

[8] EH Putra, MY Hariyawan, A Gunawan, Wireless Sensor Network for Forest Fire Detection, Telkomnika Indonesian Journal of Electrical Engineering, vol.11, no.3, 2013, pp. 563-574

[9] B,C, Villaverde, S, Rea, D, Pesch, InRout - a QoS aware route selection algorithm for industrial wireless sensor networks, Ad Hoc Networks, vol.10, no.3, 2012, pp. 458-478, https://doi.org/10.1016/j.adhoc.2011.07.015 
[10] K, Akkaya, S, Janapala, Maximizing connected coverage via controlled actuator relocation in wireless sensor and actuator networks, Computer Networks, vol. 52, 2008, pp. 2779279, https://doi.org/10.1016/j.comnet.2008.06.009

[11] Guangjie Han, Li Liu, Jinfang Jiang, Lei Shu, Gerhard Hancke, Analysis of EnergyEfficient Connected Target Coverage Algorithms for Industrial Wireless SensorNetworks, IEEE Transaction on Industrial Informatics, 2015, https://doi.org/10.1109/TII.2015.251 $\underline{3767}$

[12] I, Guneydas, K, Akkaya, A, Bicak, Actuator positioning in wireless sensor and actuator networks using matching theory, 29th IEEE International Conference on Distributed Computing Systems Workshops, Montreal, 200, 88-95.

[13] McLaughlan B, Akikaya K, Coverage-based clustering of wireless sensor and actor network, IEEE international conference on pervasive services, Istanbul, 2007, 45-54.

[14] Yan Xiaoying, Cheng Guojian, Zhou Guanwu, QPSO based actor optimal development in WSAN, Application research of computers, vol. 29, no.10, 2012, 3873-3875.

[15] Che Nan, Li Zhijun, Jiang Shouxu, Optimal actor node development strategy under realtime constrain in WSANs, Journal of software, vol.22, no.6, 2011, pp.1361-1372. https://doi.org/10.3724/SP.J.1001.2011.03826

[16] Gangyong Jia, Guangjie Han, Jinfang Jiang, Li Liu, Dynamic Adaptive Replacement Policy in Shared LastLevel Cache of DRAM/PCM Hybrid Memory for Big Data Storage, IEEE Transactions on Industrial Informatics, 2016, https://doi.org/10.1109/TII.2016.264 $\underline{5941}$

\section{Authors}

Xuejun Jiang, Wenbo Zhang*, Lincong Zhang, and Xiaobo Tan are with School of Information Science \& Engineering, Shenyang Ligong University, No.6, nanping center Road, Hunnan New District, Shenyang, Liaoning, P.R.China (e-mail: 1159365437@qq.com).

Article submitted 24 February 2017. Published as resubmitted by the authors 13 April 2017. 